\title{
Treatment of insertional Achilles tendinopathy with ultrasound-guided intrabursal retrocalcaneal hyaluronic acid injection - a prospective study
}

Omer Slevin ( $\nabla$ omerslevin@gmail.com )

Meir Medical Center https://orcid.org/0000-0002-9294-525X

David Segal

Meir Medical Center

Nissim Ohana

Meir Medical Center

Eugene Kots

Meir Medical Center

Viktor Feldman

Meir Medical Center

Meir Nyska

Meir Medical Center

Ezequiel Palmanovich

Meir Medical Center

Research article

Keywords: Achilles tendon, Insertional Achilles tendinopathy, Enthesitis, Retrocalcaneal bursitis, Hyaluronic acid, Ultrasound, Sonography

Posted Date: October 1st, 2020

DOI: https://doi.org/10.21203/rs.3.rs-32768/v1

License: (a) (1) This work is licensed under a Creative Commons Attribution 4.0 International License. Read Full License 


\section{Abstract}

\section{Background}

Insertional Achilles tendinopathy (IAT) is a chronic degenerative enthesopathy involving fibrocartilage changes that resemble osteoarthritic changes in articular cartilage. Thus, our primary goal was to evaluate the effect of hyaluronic acid (HA) injections on IAT.

Methods

Fifteen IAT ankles (14 patients) were treated with three consecutive weekly ultrasound-guided retrocalcaneal intrabursal injections of hylan G-F 20 (Synvisc $\left.{ }^{\circledR}\right)$. Patients answered the "Victorian Institute of Sport Assessment - Achilles" (VISA-A) questionnaire before every injection and on 1 month and 6 months follow-up visits. Univariate analysis was performed to identify differences in functional scores.

Results

The mean (VISA-A) score improved significantly following HA injections from $34.8 \pm 15.2$ (range, 11-63) points before the first injection to $53.6 \pm 20.9$ (range, 15-77) points after 1 month, and $50.7 \pm 18.6$ (20-75) points after 6 months. No adverse drug reactions were noted.

Conclusions

Three consecutive ultrasound-guided intrabursal retrocalcaneal HA injections were found in our cohort to be beneficial in treating IAT.

Trial registration

NCT02368561. Registered 23 February 2015. https://clinicaltrials.gov/ct2/show/NCT02368561? term=insertional+achilles\&draw $=2 \&$ rank $=2$

\section{Introduction}

Insertional Achilles tendinopathy (IAT) is a chronic degenerative enthesopathy, affecting both physically active and non-active individuals [1]. The diagnosis is mainly clinical and is based on posterior heel pain, localized to the insertion of the Achilles tendon, accompanied by local swelling and impaired function [2]. About $20 \%$ of the patients suffering from chronic Achilles overuse injury have clinical and histopathological signs of IAT [3]. The pathological process in IAT may also involve a retrocalcaneal bursitis [4]. Significant histopathological degenerative changes in IAT were found not only in the tendon, but also in the calcaneal fibrocartilage layers surrounding the distal part of the retrocalcaneal bursa [5]. These degenerative changes were found to resemble changes to articular cartilage that occur in osteoarthritis $[5,6]$. Furthermore, the inflammatory changes, such as retrocalcaneal bursitis, were 
suggested to be secondary to the fibrocartilage degeneration [6]. Those findings imply that IAT may not be merely an activity-related pathology, but rather a more complex process involving all parts of the enthesis [7-9].

The degenerative component in IAT has recently led researchers to suspect that arthritis-modifying agents, such as hyaluronic acid (HA), may be applicable in the treatment of IAT [5]. Several histological studies that investigated the effects of exogenous HA on enthesopathies have shown that HA can reduce inflammatory mediators [10], inhibit cartilage degeneration [11], and promote neovascularization and growth of new sensory nerves [12]. Due to the osteoarthritic-like changes in the fibrocartilage, mainly in the calcaneal posterior wall, we hypothesized that retrocalcaneal intrabursal HA injections would have a beneficial effect on IAT patients. The primary goal of this study was to evaluate the effect of this treatment modality for IAT. The secondary goal was to assess whether IAT can be categorized according to four distinct sonographic findings and to correlate these findings with the treatment outcomes.

\section{Materials And Methods}

We conducted a prospective, uncontrolled, single-center study between the years 2015 and 2017. Fifteen IAT cases in 14 patients were treated by three consecutive weekly ultrasound-guided retrocalcaneal intrabursal injections of high-molecular hylan G-F 20 (Synvisc ${ }^{\circledR}$; Genzyme Biosurgery, Genzyme Corporation, NJ, USA). Patients were followed in a specialized foot and ankle clinic for at least 6 months. An ethical approval was obtained from the local ethical committee (0082-14 MMC). All procedures performed were in accordance with the ethical standards of the institutional and/or national research committee and with the 1964 Declaration of Helsinki and its later amendments or comparable ethical standards. All participants signed an informed consent form.

Inclusion criteria were a diagnosis of chronic IAT that was established by a foot and ankle orthopedic surgeon, and a rheumatology clinic assessment in which a systemic arthropathy was ruled out. The diagnosis was based on a history of pain at the calcaneal insertion of the Achilles tendon and a physical examination that revealed tenderness in the calcaneal insertion of the Achilles tendon. A mandatory radiographic work-up included an Magnetic resonance imaging (MRI) study of the relevant foot and ankle, by which the soft tissue was evaluated for both insertional and peritendinous edema. Exclusion criteria included previous surgical treatment such as retrocalcaneal osteotomy, retrocalcaneal injection of platelet-rich plasma (PRP) or steroids during the 6 months prior to the enrollment, previous two or more steroids injection, a history of Achilles tendon rupture or a previous calcaneal fracture.

During the first visit to the clinic, detailed demographic data, medical history and previous treatment modalities were documented. A physical examination was performed by one of the two abovementioned authors and the patients were asked to fill out the "VISA-A" questionnaire (see below). All the injections were performed by one experienced radiologist by the following technique: The patients were placed in a prone position with the knee slightly flexed. The ultrasound probe was placed transverse to the Achilles tendon and the retrocalcaneal bursa. In the transverse sonographic view, the hypoechoic area (the 
retrocalcaneal bursa) was situated between the Achilles tendon and the calcaneus (Figure 1). During the first test, four different areas were examined to characterize the pathology process: A swelling and irregularity in the retrocalcaneal bursa, representing an inflammatory process, an irregularity in the posterior calcaneal wall that represents arthritis, an irregularity in the Achilles tendon that represents tendinitis, and an enthesitis reflected as an irregularity in the Achilles tendon insertion (Figure 2).

Next, a 21-gauge needle was inserted from the lateral side into a point just anterior to the Achilles tendon and a volume of $1-2 \mathrm{ml}$ of Synvisc $\AA$ was injected into the retrocalcaneal bursa. The study product is a sterile, nonpyrogenic, elastoviscous fluid containing hylans, a derivative of hyaluronan, the sodium salt of hyaluronic acid. Each $2 \mathrm{ml}$ glass syringe of Synvisc ${ }^{\circledR}$ contains $16 \mathrm{mg}$ of hylan G-F 20 (cross-linked hylan polymers; hylan A and B), $17 \mathrm{mg}$ sodium chloride, $0.32 \mathrm{mg}$ disodium hydrogen phosphate, and $0.08 \mathrm{mg}$ sodium dihydrogen phosphate monohydrate. The hyaluronan is extracted from chicken combs and the purified material has an average molecular weight of $6,000 \mathrm{kDa}$. The dose was adapted according to intrabursal pressure and the procedure was terminated when the retrocalcaneal bursa appeared brimful on the sonographic view. The patients were asked to avoid vigorous exercise during the first two days following the injection. Thereafter, the patients were instructed to try to return to full activity.

For outcome evaluation, we used the Victorian Institute of Sport Assessment - Achilles (VISA-A) questionnaire [13]. This questionnaire was designed specifically to assess the severity of Achilles tendinopathy. The questionnaire is a self-administered form that evaluates pain, function and activity level [13] and has been commonly used to monitor outcomes after different treatment modalities for Achilles tendinopathy. It was previously found to be valid, reliable and clinically relevant $[13,14]$. The scores range from 0 to 100 points, with higher scores indicative of better function. The minimum clinically important difference (MCID), which is defined as the smallest change on a scale that would be considered important to a patient, has been estimated to be between 6 and 12 points $[15,16]$.

A detailed history of daily and sport activities, physical examination results and physical adverse events were assessed 1 month and 6 months following the primary injection. The patients were asked to answer the VISA-A questionnaire before every injection and on each follow-up visit.

\subsection{Statistical analysis}

Descriptive statistics included means, medians, ranges, and standard deviations calculated for the demographic variables and the VISA-A scores. For evaluation of the difference from the baseline at 1 month and 6 months, we used a two-tailed Mann-Whitney $U$ test for nonparametric variables. The level of significance was set at $p=0.05$. Analyses were performed using IBM SPSS-25 (Chicago, IL) statistical package software.

\section{Results}

The mean age of patients, 12 males and 3 females, was $53.7 \pm 11.5$ (range, 32-61) years. On baseline imaging, all patients had at least one of four sonographic features: enthesitis reflected as an irregularity 
in the Achilles tendon insertion ( $n=15)$, Achilles tendon irregularity $(n=12)$, retrocalcaneal bursitis $(n=5)$ and an irregularity in the posterior calcaneal wall $(n=5)($ Table 1$)$.

At follow-up, two patients did not experience any functional improvement. Six patients reported a significant pain relief, mainly during daily life activity with various levels of pain and functional improvement in low-intensity physical activity. One patient reported a significant pain relief and functional improvement during the first 5 months, followed by a significant functional decline. Analysis of the VISA-A questionnaires showed that the improvement in the mean functional score following three consecutive HA injections was statistically significant. The mean VISA-A score was $34.8 \pm 15.2$ (range, 11-63) points before the first injection, $53.6 \pm 20.9$ (range, 15-77) points after 4 weeks, and $50.7 \pm 18.6$ (range, 20-75) points after 6 months (Table 2). Two patients underwent further surgical treatment due to insufficient improvement. No adverse drug reaction or infection following injection were noted in any of the patients. We did not find a correlation between specific sonographic features and the functional level at presentation or following treatment, as indicated by the VISA-A scores $(P>0.05)$.

\section{Discussion}

The most important finding of the present study is that in most of the patients who participated in the study, retrocalcaneal intrabursal injections of HA have led to clinical improvement in IAT related symptoms. The functional improvement following HA injection might be related to the complex histopathology of IAT, as indicated by the latest findings showing that the degenerative changes are not only in the tendon, but also in the calcaneal fibrocartilage layers surrounding the distal part of the retrocalcaneal bursa [5].

Until recently, the main etiology of IAT was considered to be overuse in patients with poor physical training habits, which include repetitive traction force [2]. However, it was lately suggested that the fibrocartilage degenerative lesions might be the result of a "stress-shielding" reaction to long periods of less tensile load in an anterior part of the enthesis [2,7-8]. Moreover, the degenerative changes, particularly fissuring and chondrocyte clustering, were found to resemble osteoarthritic articular cartilage changes [5, 6] and the severity of these histological changes, as indicated by an osteoarthritic grading system, correlated with the degree of functional impairment [5]. Those findings imply that IAT may be a complex pathological process $[7,8]$ involving the periosteal and sesamoid fibrocartilages, the retrocalcaneal bursa and the fat pad [9], which are all parts of the enthesis.

Overall, the majority of patients with IAT are successfully treated non-operatively, with activity modification, non-steroidal anti-inflammatory medications and physical therapy with eccentric training of gastrocnemius and soleus muscles [2]. However, the stretching exercises that are very successful in noninsertional Achilles tendinopathy were found to be less effective in IAT [17].

A variety of local injection-based therapies that deliver a drug directly to the damaged tendon or to the retrocalcaneal bursa were described, including PRP and autologous blood and corticosteroid. Yet, a recently published review by the Cochrane library found that the currently available evidence is 
insufficient to support the routine use of these modalities [18]. When the non-operative management fails to improve symptoms, a surgical approach may be indicated. The surgical treatment includes a debridement of the ill portion of the tendon, an excision of the retrocalcaneal bursa and a resection of the calcaneal posterior wall or superior prominence [2,5].

Hyaluronic acid is a polysaccharide with relatively high molecular weight (between $100 \mathrm{kDa}$ and $10 \mathrm{MDa}$ ) that is found mostly in the extracellular matrix of numerous tissue types in the human body [19]. The primary role of $\mathrm{HA}$ in connective tissues is to increase the viscoelasticity by binding water and to lubricate the synovial joints $[19,20]$. Currently, intra-articular injection of HA is mainly used as a viscosupplementation as part of the conservative treatment of knee osteoarthritis [21]. Recently, it has been proposed to utilize the viscoelastic and anti-inflammatory properties of HA for the treatment of different tendinopathies and enthesopathies $[19,20]$. Although in vitro and animal studies produced encouraging results, including better tendon architectural organization, reduced adhesions and enhanced tendon gliding, only a limited number of clinical studies were published on the use of HA for tendinopathies, mainly regarding rotator cuff tendinopathy and elbow epicondylitis [19].

To date, much has been published on different conservative treatments for non-insertional Achilles tendinopathy, including PRP injection [16], high-energy extracorporeal shock wave therapy [22], topical glyceryl trinitrate patches [23], cryotherapy [24], laser therapy and eccentric exercise [25]. However, less evidence is available regarding the non-operative treatment of IAT [26]. Reports on treatment outcomes of injection therapies for IAT are even scarcer. Ohberg et al. prospectively analyzed 11 patients, who were treated with a sclerosing agent (polidocanol), which was injected against the local neovascularization found in the distal tendon portion [27]. They found that the mean Visual Analogue Scale (VAS) pain score decreased by 5.9 at 8 months follow-up, and eight out of 11 (73\%) patients found the treatment satisfactory. Ryan et al. retrospectively analyzed 22 patients treated with ultrasound-guided hyperosmolar dextrose $(20 \mathrm{mg} / \mathrm{mL})$ injections [29]. The mean VAS on daily activity decreased by 4.1 points $(p=0.001)$ at a follow-up of 28.6 months. To our knowledge, despite those encouraging preliminary results, no further studies were published on the use of similar agents for the treatment of IAT. Rompe et al. compared extracorporeal shockwave therapy (ESWT) with an eccentric training regime in a randomized controlled trial and found a 10-point increase in the VISA score in patients treated with eccentric training and a 27-point increase in patients treated with ESWT [30]. In light of these findings, the mean improvement produced by the modality offered in the current study, namely an increase of 15.9 points in the VISA-A score at 6 months follow-up with no adverse effects, seems to be a legitimate treatment option in IAT.

The wide range of improvement on the VISA-A scale ( 6 - 44 points) on the last follow-up, along with the diversity in imaging findings, raise the suspicion that IAT may in fact be a combination of several pathologies. Distinguishing between those entities, especially in terms of therapeutic implications, has barely been discussed in the literature. Dijk et al. reviewed the terminology for Achilles tendon injuries and proposed new definitions, based on the anatomic location, symptoms, clinical findings and histopathology [28]. The authors have made a clear distinction between mid-portion Achilles 
tendinopathy, Achilles paratendinopathy, insertional Achilles tendinopathy, retrocalcaneal bursitis and superficial calcaneal bursitis. In their systematic review on the treatment for IAT, Wiegerinck et al. hypothesized that patients with IAT and bony disorder located at the calcaneal insertion of the Achilles tendon may have different treatment outcomes when compared with IAT patients without bony pathology [26]. Five of the patients in our series had radiographic findings compatible with retrocalcaneal bursitis. The inflammatory characteristics of retrocalcaneal bursitis, combined with the degenerative process found in fibrocartilage and tendon tissues, implies that IAT has a complex pathogenesis. Since the major advantage of $\mathrm{HA}$ injection is improving the viscoelasticity and affecting degenerative cartilage tissue, the impact of HA injection for IAT was expected to be greater in patients with an irregularity in the posterior calcaneal wall, but without retrocalcaneal bursitis. This assumption could not be proved in this cohort of patients.

The present prospective pilot case series bears a number of limitations. Clearly, as the study did not include a control group, the results should be considered only in a descriptive manner. Moreover, the number of participants is insufficient for drawing a clear therapeutic recommendation or for high statistical power. Nevertheless, the study was based on a biological rationale, included an advanced imaging modalities-based diagnosis and yield promising results. We therefore believe that our results warrant a larger scale investigation of this innovative treatment option.

\section{Conclusion}

In the current prospective study, ultrasound-guided, intrabursal, retrocalcaneal HA injections were found to be a highly promising treatment for IAT.

\section{Abbreviations}

IAT: Insertional Achilles tendinopathy, HA: hyaluronic acid, MRI: Magnetic resonance imaging, PRP: platelet-rich plasma, VISA-A: Victorian Institute of Sport Assessment - Achilles, MCID: minimum clinically important difference, VAS: Visual Analogue Scale, ESWT: extracorporeal shockwave therapy

\section{Declarations}

\section{Ethics approval and consent to participate}

The study described in the article was approved by the Meir Medical Center IRB committee.

\section{Consent for publication}

Not applicable.

\section{Availability of data and materials}

The datasets used and/or analyzed during the current study are available 
from the corresponding author on reasonable request.

\section{Competing interests}

The authors declare that they have no competing interests.

\section{Funding}

This research did not receive any specific grant from funding agencies in the public, commercial, or notfor-profit sectors.

\section{Authors' contributions}

OS and VF collected the data. EK performed the sonography and the injections and analyzed the images. $\mathrm{OS}, \mathrm{DS}$, and EP were the major contributors in writing the manuscript. NO and MN reviewed the manuscript. All authors read and approved the final manuscript.

\section{References}

[1] Myerson MS, McGarvey W. Disorders of the Achilles tendon insertion and Achilles tendinitis. Instr Course Lect. 1999;48:211-218.

[2] Sayana MK, Maffulli N. Insertional achilles tendinopathy. Foot Ankle Clin. 2005;10(2):309-320. doi:10.1016/j.fcl.2005.01.010.

[3] Astrom M, Rausing A. Chronic Achilles tendinopathy. A survey of surgical and histopathologic findings. Clin Orthop Relat Res. 1995;(316):151-164.

[4] Paavola M, Orava S, Leppilahti J, Kannus P, Järvinen M. Chronic Achilles tendon overuse injury: complications after surgical treatment. An analysis of 432 consecutive patients. Am J Sports Med. 2000;28(1):77-82.

[5] Palmanovich E, Oshri Y, Brin YS, Edelshtein E, Nyska M, Hetsroni I. Insertional Achilles tendinopathy is associated with arthritic changes of the posterior calcaneal cartilage: a retrospective study. $J$ Foot Ankle Res. 2015;8(1):44. doi:10.1186/s13047-015-0103-8.

[6] Rufai A, Ralphs JR, Benjamin M. Structure and histopathology of the insertion region of the human achilles tendon. J Orthop Res. 1995;13:585-593.

[7] Lyman J. Strain Behavior of the Distal Achilles Tendon: Implications for Insertional Achilles Tendinopathy. Am J Sports Med. 2004;32(2):457-461. doi:10.1177/0095399703258621.

[8] Maganaris CN, Narici M V, Almekinders LC, Maffulli N. Biomechanics and pathophysiology of overuse tendon injuries: ideas on inser...: EBSCOhost. 2004;34(14):1005-1017. 
[9] Milz S, Rufai A, Buettner A, Putz R, Ralphs JR, Benjamin M. Three-dimensional reconstructions of the Achilles tendon insertion in man. J Anat. 2002;200(2):145-152. doi:10.1046/j.0021-8782.2001.00016.x.

[10] Tamoto K, Nochi H, Tokumitsu Y. High molecular weight hyaluronic acids inhibit interleukin-1-induced prostaglandin E2 generation and prostaglandin E2-elicited cyclic AMP accumulation in human rheumatoid arthritic synovial cells. Japanese J Rheumatol. 5(3):227-236.

[11] Kikuchi T, Shimmei M. Effects of hyaluronan on proteoglycan metabolism of rabbit articular chondrocytes in culture. Japanese J Rheumatol. 5(3):207-215.

[12] Tang T, Muneta T, Sekiya I. Fibrous change of the infrapatellar fat pad due to strenuous running exercise and its treatment with intraarticular hyaluronan injection in a rat model. J Med Dent Sci. 2008;55(1):163-173.

[13] Robinson JM, Cook JL, Purdam C, et al. The VISA-A questionnaire: a valid and reliable index of the clinical severity of Achilles tendinopathy. Br J Sports Med. 2001;35(5):335-341.

doi:10.1136/bjsm.35.5.335.

[14] Iversen JV, Bartels EM, Langberg H. the Victorian Institute of Sports Assessment - Achilles Questionnaire (Visa-a) - a Reliable Tool for Measuring Achilles Tendinopathy. Int J Sports Phys Ther. 2012;[23](1):76-84.

[15] McCormack J, Underwood F, Slaven E, Cappaert T. the Minimum Clinically Important Difference on the Visa-a and Lefs for Patients With Insertional Achilles Tendinopathy. Int J Sports Phys Ther. 2015;10(5):639-644.

[16] Vos RJ De, Weir A, Schie HTM Van, Weinans H, Page P. Platelet-Rich Plasma Injection for Chronic Achilles Tendinopathy. Jama. 2010;303(2):144-149. doi:10.1001/jama.2009.1986.

[17] Fahlström M, Jonsson P, Lorentzon R, Alfredson H. Chronic Achilles tendon pain treated with eccentric calf-muscle training. Knee surgery, Sport Traumatol Arthrosc. 2003;11(5):327-333. doi:10.1007/s00167-003-0418-z.

[18] Kearney RS, Parsons N, Metcalfe D, et al. Injection therapies for Achilles tendinopathy (Review ) Injection therapies for Achilles tendinopathy. 2015;(5). doi:10.1002/14651858.

[19] Kaux J-F, Samson A, Crielaard J-M. Hyaluronic acid and tendon lesions. Muscles Ligaments Tendons J. 2015;5(4):264-269. doi:10.11138/mltj/2015.5.4.264.

[20] Kumai T, Muneta T, Tsuchiya A, et al. The short-term effect after a single injection of high-molecularweight hyaluronic acid in patients with enthesopathies (lateral epicondylitis, patellar tendinopathy, insertional Achilles tendinopathy, and plantar fasciitis): A preliminary study. J Orthop Sci. 2014;19(4):603611. doi:10.1007/s00776-014-0579-2. 
[21] Bannuru RR, Natov NS, Dasi UR, Schmid CH, McAlindon TE. Therapeutic trajectory following intraarticular hyaluronic acid injection in knee osteoarthritis - meta-analysis. Osteoarthr Cartil. 2011;19(6):611619. doi:10.1016/j.joca.2010.09.014.

[22] Furia JP. High-energy extracorporeal shock wave therapy as a treatment for chronic noninsertional Achilles tendinopathy. Am J Sports Med. 2008;36(3):502-508. doi:10.1177/0363546507309674.

[23] Kane TPC, Ismail M, Calder JDF. Topical glyceryl trinitrate and noninsertional Achilles tendinopathy: a clinical and cellular investigation. Am J Sports Med. 2008;36(6):1160-1163.

doi:10.1177/0363546508314423.

[24] Knobloch K, Grasemann R, Spies M, Vogt PM. Midportion achilles tendon microcirculation after intermittent combined cryotherapy and compression compared with cryotherapy alone: a randomized trial. Am J Sports Med. 2008;36(11):2128-2138. doi:10.1177/0363546508319313.

[25] Stergioulas A, Stergioula M, Aarskog R, Lopes-Martins RAB, Bjordal JM. Effects of low-level laser therapy and eccentric exercises in the treatment of recreational athletes with chronic achilles tendinopathy. Am J Sports Med. 2008;36(5):881-887. doi:10.1177/0363546507312165.

[26] Wiegerinck JI, Kerkhoffs GM, van Sterkenburg MN, Sierevelt IN, van Dijk CN. Treatment for insertional Achilles tendinopathy: A systematic review. Knee Surgery, Sport Traumatol Arthrosc. 2013;21(6):13451355. doi:10.1007/s00167-012-2219-8.

[27] Ohberg L, Alfredson H. Sclerosing therapy in chronic Achilles tendon insertional pain-results of a pilot study. Knee Surg Sports Traumatol Arthrosc. 2003;11(5):339-343. doi:10.1007/s00167-003-0402-7.

[28] van Dijk CN, van Sterkenburg MN, Wiegerinck JI, Karlsson J, Maffulli N. Terminology for Achilles tendon related disorders. Knee Surgery, Sport Traumatol Arthrosc. 2011;19(5):835-841. doi:10.1007/s00167-010-1374-z.

[29] Ryan M, Wong A, Taunton J. Favorable outcomes after sonographically guided intratendinous injection of hyperosmolar dextrose for chronic insertional and midportion achilles tendinosis. Am J Roentgenol. 2010;194(4):1047-1053. doi:10.2214/AJR.09.3255.

[30] Rompe JD, Furia J, Maffulli N. Eccentric Loading Compared with Shock Wave Treatment for Chronic Insertional Achilles Tendinopathy. A Randomized, Controlled Trial. J Bone Jt Surg. 2008;90(1):52. doi:10.2106/JBJS.F.01494.

\section{Tables}

Table 1. Demographic, sonographic and clinical findings of the study cohort 


\begin{tabular}{|ll|}
\hline & \\
\hline Age & $53.7 \pm 11.5$ years (range, 32-61) \\
\hline Male: Female & $12: 3$ \\
\hline Unilateral : Bilateral IAT & $13: 1$ \\
\hline Achilles tendinopathy & $12 / 15$ \\
\hline Retrocalcaneal bursitis & $5 / 15$ \\
\hline Posterior calcaneal wall irregularity & $5 / 15$ \\
\hline Mean baseline VISA-A score (range) & $34.8 \pm 15.2(11-63)$ \\
\hline
\end{tabular}

Table 2. Treatment results according to the VISA-A score

Mean VISA-A score after 1 month $53.6 \pm 20.9(15-$ 77)

Mean VISA-A score after 6 months $50.7 \pm 18.6(20-$ 75)

Mean VISA-A score after 6 months in IAT with bursitis $44 \pm 13.3(20-48)$

Mean VISA-A score after 6 months in IAT with irregularity in the posterior calcaneal wall

$63.6 \pm 17.2(48-$ 75)

\section{Figures}
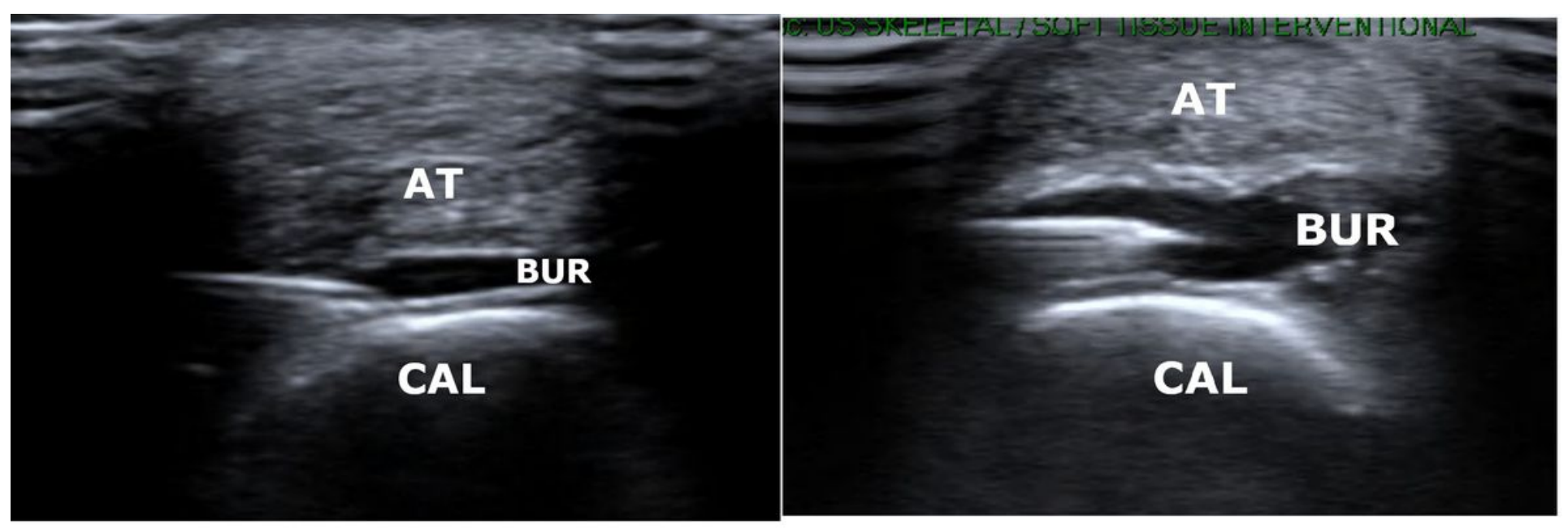

A

B 
Sonography images of the retrocalcaneal intrabursal injection of HA. Abbreviations: AT, Achilles tendon; BUR, retrocalcaneal bursa; CAL, calcaneus.
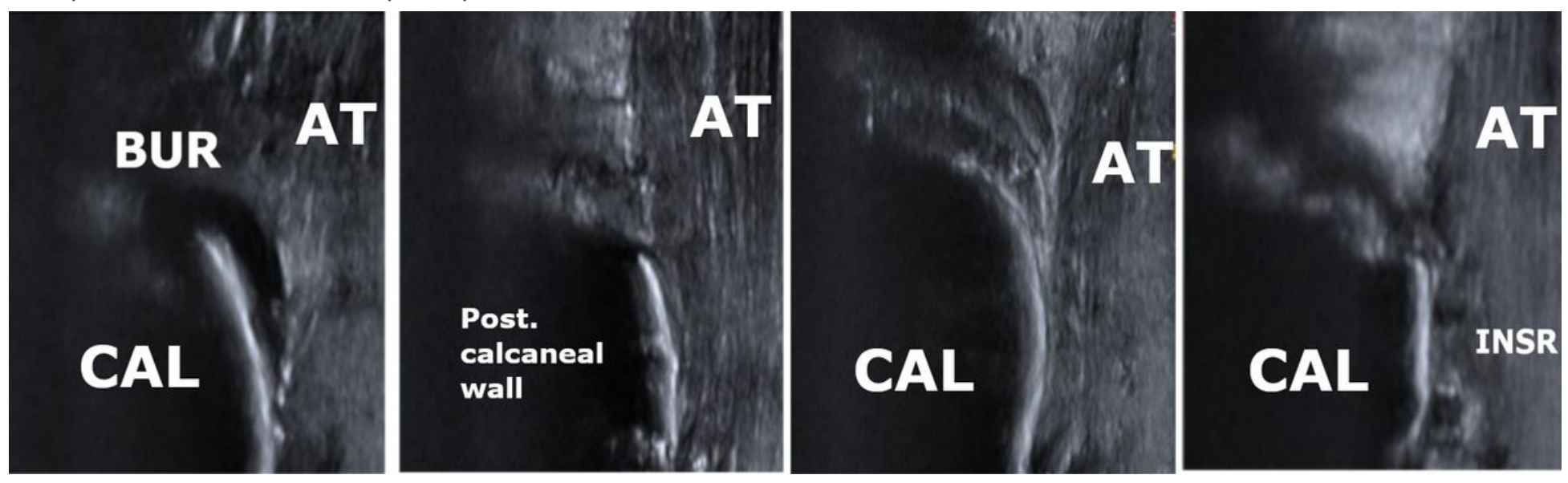

Figure 2

The sonographic pathological patterns in IAT.\&(A) Retrocalcaneal bursitis. (B) Posterior calcaneal wall irregularity. (C) Enthesitis. (D) Achilles tendon irregularity. Abbreviations: AT, Achilles tendon; BUR, retrocalcaneal bursa; CAL, calcaneus; INSR, Achilles tendon insertion. 